\title{
Efficacy and Safety of Lianhua Qingke Tablets in the Treatment of Mild and Common-Type COVID-19: A Randomized, Controlled, Multicenter Clinical Study
}

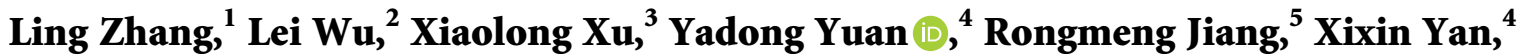 \\ Xin Zhang, ${ }^{1}$ Yong Gao, ${ }^{1}$ Huanxia Shang, ${ }^{1}$ Bo Lian, ${ }^{3}$ Jing Hu $\mathbb{D}^{3},{ }^{3}$ Jianqiang Mei $\mathbb{D},{ }^{2}$ \\ Shucai Wu $\mathbb{D}^{1}$, and Qingquan Liu $\mathbb{D}^{3}$ \\ ${ }^{1}$ Hebei Chest Hospital, Shijiazhuang 050041, China \\ ${ }^{2}$ Hebei Hospital of Traditional Chinese Medicine, Shijiazhuang 050011, China \\ ${ }^{3}$ Beijing Hospital of Traditional Chinese Medicine, Capital Medical University, Beijing 100010, China \\ ${ }^{4}$ The Second Hospital of Hebei Medical University, Shijiazhuang 050000, China \\ ${ }^{5}$ Beijing Ditan Hospital, Capital Medical University, Beijing 100015, China
}

Correspondence should be addressed to Jianqiang Mei; mjq1000000@sina.com, Shucai Wu; shucaiwu2009@163.com, and Qingquan Liu; liuqingquan_2003@126.com

Received 2 August 2021; Revised 21 September 2021; Accepted 8 January 2022; Published 10 February 2022

Academic Editor: Wansu Park

Copyright (c) 2022 Ling Zhang et al. This is an open access article distributed under the Creative Commons Attribution License, which permits unrestricted use, distribution, and reproduction in any medium, provided the original work is properly cited.

\begin{abstract}
Background. Lianhua Qingke (LH) tablets is an effective traditional Chinese medicine against various viral infections, especially in relieving coughing. However, its effects on COVID-19 are unknown. Methods. To examine the therapeutic effectiveness of LH tablets in COVID-19 patients with mild and common types, a randomized, multicenter, controlled study was carried out. COVID-19 cases were randomized to undergo routine treatment with or without LH tablets ( 4 tablets, three times a day) for 14 days. The primary endpoints were the rate of achieving clinical symptom resolution and the corresponding time. Results. There were 144 participants in the full analysis set (72 each in the LH and control groups). The LH group participants had elevated symptom alleviation rate at 14 days compared with control cases (FAS: $98.61 \%$ vs. $84.72 \%, p=0.0026$ ). In comparison with control group participants, the LH group participants had reduced median time to clinical symptom alleviation (median: 4 vs. 7 days, $p<0.0001)$. Higher resolution rates of coughing $(98.44 \%$ vs. $84.51 \%, p=0.0045)$ and expectoration $(100 \%$ vs. $82.35 \%$, $p=0.0268$ ) were observed in the LH group. Times to recovery of fever (median: 2 vs. 3 days, $p=0.0007$ ), coughing (median: 4 vs. 7 days, $p<0.0001$ ), and expectoration (median: 3 vs. 6 days, $p<0.0001$ ) were also notably shorter in the LH group. Moreover, the LH group had elevated improvement rates in chest computed tomography signs (FAS: $86.11 \%$ vs. $72.22 \%$, $p=0.0402$ ) and clinical cure at day 28 (FAS: $83.33 \%$ vs. $68.06 \%, p=0.0326$ ). However, no differences were found in the laboratory test and viral assay. Serious adverse events were not detected. Conclusion. These preliminary findings indicate LH tablets may be effective in symptomatic COVID-19, especially in relieving coughing. This trial was registered in Chinese Clinical Trial Registry (ChiCTR2100042069).
\end{abstract}

\section{Introduction}

In December 2019, coronavirus disease 2019 (COVID-19), resulting from infection by severe acute respiratory syndrome coronavirus 2 (SARS-CoV-2), emerged as a new pandemic that has continued to pose a severe threat to the global community [1]. As of April 2021, more than 146.8 million confirmed cases and 3.1 million deaths have been reported across 188 countries (World Health Organization, WHO). It has been reported that COVID-19 vaccines may have a limited effect in combating SARS-CoV-2 spread and protecting from symptomatic COVID-19 [2]. A recent solidarity trial by the WHO also reported that four most promising antiviral drugs, including remdesivir, 
hydroxychloroquine, lopinavir, and interferon regimens, are minimally effective in severe COVID-19 [3]. Therefore, there is still no specific remedy available to infected patients.

Coughing is considered a major clinical manifestation in COVID-19 patients. Pathological anatomy showed mucus and mucus-related thrombosis in the bronchial cavity, serous, fibrin exudate, and hyaline membrane formation in the alveolar cavity [4]. These viscous exudates block the airway, which seriously affects the ventilation function of the lung, reduces the efficacy of oxygen therapy and mechanical ventilation, and even causes asphyxial death [5]. In addition, coughing is a stigma for patients recovering from COVID19 , as a common post-COVID-19 syndrome symptom [6]. Based on reports and feedback from patients, many individuals are with post-COVID syndrome symptoms such as coughing dread reinfection, staying away from relatives and friends, and/or have trouble resuming work [7]. Therefore, taking effective measures and administering drugs to ensure airway patency and improve lung ventilation function has become an important way to improve the cure rate of COVID-19 cases. However, the current lack of effective anticoronavirus drugs is compounded by the scarcity of effective drugs for the treatment of the respiratory tract, especially targeting small airway mucus or sputum embolism [6]. Therefore, it is urgent to develop effective drugs to relieve clinical symptoms, especially cough and sputum.

LH tablets is a marketed patented Chinese medicine drug designed for treating respiratory diseases, especially those accompanied by cough and sputum. It was approved by the State Food and Drug Administration (SFDA No. 2010L00120) in 2010 and underwent a phase III clinical trial in 2014 with confirmed efficiency. LH tablets is mainly composed of thirteen herbs, including Mahuang (stem, Herba Ephedra), Sangbaipi (root bark, Morus Alba L.), Shigao (Gypsum fibrosum), Huangqin (root, Scutellaria baicalensis), Kuxingren (semen, armeniacae amarum), Lianqiao (fruit, Forsythia suspensa), Banxia (stem, Pinellia ternate), Zhebeimu (bulb, Fritillaria thunbergii), Qianhu (root, Peucedanum praeruptorum), Niubangzi (fruit, Fructus arctii), Jinyinhua (flos, Lonicera japonica Thunb.), Dahuang (root, Rhei Radix et Rhizoma), and Chenpi (pericarpium, Citrus reticulata Blanco.). The main function of $\mathrm{LH}$ is stopping cough. The indications of LH include acute tracheitis and bronchitis-caused cough and headache [8].

Considering COVID-19 symptoms and LH tablet indications, we hypothesized that LH might be beneficial for cases showing upper respiratory symptoms. Here, we conducted a randomized controlled study, aiming to examine the effectiveness of LH tablets in COVID-19.

\section{Patients and Methods}

2.1. Study Design and Patients. The current randomized, controlled, multicenter study examined COVID-19 cases diagnosed based on the Diagnosis and Treatment Program for Novel Coronavirus Infection Pneumonia (Trial Eighth Edition) released by the National Health Commission of China. This trial was planned and prepared since January 1, 2021, and started enrollment from January 14 to March 31, 2021. This study was performed in Thoracic Surgery Department, Tuberculosis Department, and Department of Neurology of the Hebei Chest Hospital and Second Hospital of Xingtai and Harbin Chest Hospital. During the outbreak of COVID-19 in Shijiazhuang, the whole Hebei Chest Hospital and Second Hospital of Xingtai were changed into COVID-19-designated hospitals, which only received and cured COVID-19 patients. This trial was approved by the Ethics Committee of Hebei Province Chest Hospital and was performed according to the GCP guidelines and the Declaration of Helsinki. Each patient or designated legal representative provided signed informed consent. This trial was registered in Chinese Clinical Trial Registry (ChiCTR2100042069).

Inclusion criteria were as follows: confirmed COVID-19 by virus testing; $\geq 18$ years of age; fever, cough, expectoration, chest tightness, polypnea, and/or dyspnea at enrollment; and informed consent provided. Exclusion criteria were as follows: overt bacterial infection in the respiratory tract resulting from common pathologies, including primary immunodeficiency disease, acquired immunodeficiency syndrome, congenital respiratory malformation, congenital heart disease, gastroesophageal reflux disease, and abnormal lung development; asthma treated daily, chronic airway disease, respiratory bacterial infections (e.g., purulent tonsillitis), acute tracheobronchitis, sinusitis, otitis media, and further respiratory tract pathologies potentially affecting the trial's data analysis; common pulmonary diseases (e.g., severe pulmonary interstitial lesions and bronchiectasis) confirmed by chest CT; severe pneumonia requiring ventilator use; previous or present diseases potentially affecting trial participation or influencing study outcome, based on the investigator's judgment; pregnancy or lactation in women; participation in a clinical study in the past 3 months; and history of allergy to $\geq 2$ drugs or foods or known allergy to the drug's constituents.

2.2. Clinical Classification. Patients were classified into mild, common, and severe cases. Mild: confirmed cases with fever (measured or subjective) or respiratory symptoms. Moderate: confirmed cases with fever (measured or subjective) or respiratory symptoms and radiographic evidence of pneumonia. Severe: confirmed cases with evidence including fever, respiratory symptoms, radiographic evidence of pneumonia, and at least one of the following symptoms: respiratory distress, $\mathrm{RR} \geq 30$ times/min; resting state, mean oxygen saturation $\leq 93 \%$; and $\mathrm{PaO}_{2} / \mathrm{FiO}_{2} \leq 300 \mathrm{mmHg}$ $(1 \mathrm{mmHg}=0.133 \mathrm{kPa})$.

2.3. CT Examination. The evaluation of CT chest film is to examine the lung CT imaging of patients by experienced radiologists. The improvement of chest CT imaging refers to the following: reduction of infiltration area, reduced abnormal area of radiology ground, alleviated glass opacity, and reduced nodule density.

2.4. Randomization and Grouping. A randomized, openlabel, blank-controlled, multicenter trial was performed to estimate the efficacy and safety of LH tablets in mild and 
common-type COVID-19 cases. A total of 160 random numbers with block 4 were generated with SAS 9.4 (SAS Institute, USA). The computer-based 1:1 block randomization method was utilized to assign cases to the treatment and control groups, respectively. Consecutive patients were randomly recruited by attending clinicians until reaching the total amounts of cases assigned to that particular site. All attending clinicians have obtained the qualification of clinical trial from State Drug Administration.

2.5. Treatments. For routine management (control group), the totality of treatments followed the "Diagnosis and Treatment Program for Novel Coronavirus Infection Pneumonia (Trial Eighth Edition):" supportive oxygen therapy, administration of antivirals, and symptom management (Supplementary Table 1). In the LH treatment group, the patients received routine treatment and $\mathrm{LH}$ administration (4 tablets, thrice daily) for 14 days. LH tablets (Batch: A2005001) were provided and qualified by the Shijiazhuang Yiling Pharmaceutical Co., Ltd.

2.6. Endpoints. The primary endpoints were the rate of achieving clinical symptom resolution and the corresponding time within 14 treatment days. Clinical symptom resolution was reflected by the disappearance of major symptoms, including fever, cough, expectoration, chest tightness, polypnea, and dyspnea. Secondary endpoints included the rate of single symptom disappearance, time to major symptom disappearance, changes in color, sputum quality and amounts in cases showing expectoration, changes in oxygenation index (OI), aggravation rate within the treatment period (according to severe or critical illness definitions), rate of CT improvement, disease recovery rate, and time to and rate of the coronavirus test turning negative.

2.7. Safety. Adverse events (AEs), i.e., adverse medical events occurring during the whole trial, with or without causal association with the study drug, were recorded.

2.8. Statistical Analysis. Two-sided $p<0.05$ indicated statistical significance, unless otherwise specified. Count data were presented as number or ratio; measurement data were presented as mean and standard deviation (SD) or range, and nonnormally distributed data were presented as median and 25th and 75th quartiles. Time-event data were described by median and $95 \%$ confidence interval (CI), with 25 th and 75 th quartiles. The $t$-test or Wilcoxon rank sum test was used for the comparison of quantitative data between groups. The chi-square test or exact probability test was employed for comparing categorical data. The Wilcoxon rank sum test or $\mathrm{CMH}$ test was used for grade data. The log-rank test was employed for time-event data.

To evaluate the main efficacy, the superiority test was used to compare the treatment and control groups, followed by the Newcombe-Wilson test. The log-rank test was performed to compare both groups for recovery time. Descriptive statistics of adverse events that occurred in this study were performed, and the numbers and percentages of patients with adverse events and adverse reactions in the treatment and control groups during the study period were provided. AEs' incidence rates between groups were compared by the $\chi^{2}$ test or Fisher exact probability test. SAS 9.4 (SAS Institute, USA) was utilized for statistical analysis.

\section{Results}

3.1. Characteristics of Patients. The flowchart of the trial is shown in Figure 1. A total of 144 COVID-19 cases were included and randomized in this study from January 12, 2021 to March 31, 2021, with 72 each in the control and LH treatment groups. In the end, 144 patients were included in this trial. Both groups were comparable in age, sex, temperature at onset (Table 1), and clinical characteristics (Table 2).

3.2. Primary Endpoints. The symptom recovery rate at 14 days was elevated in the LH treatment group compared with the control group (FAS: $98.61 \%$ vs. $84.72 \%$, 95\% CI: 4.98-24.00, $p=0.0026$; PPS same as FAS) (Figure 2(a)). In addition, median time to symptom resolution was also detected in the LH treatment group in comparison with control cases (FAS: 4 days vs. 7 days, $\mathrm{HR}=0.47, p<0.0001$ ) (Figure 2(b)).

3.3. Secondary Endpoints. Compared with the control group, the recovery rate of coughing at 14 days was higher in the treatment group (FAS: $98.44 \%$ vs. $84.51 \%$, 95\% CI: 4.46-24.17, $p=0.0045$ ) (Figure 3(a)). The recovery rate of expectoration was also elevated in the treatment group (FAS: $100 \%$ vs. $82.35 \%, \quad 95 \% \quad$ CI: $2.71-33.52, \quad p=0.0268)$ (Figure 3(b)). By analyzing the time to achieving clinical symptom recovery, shorter times of cough (FAS: 4 days vs. 7 days, $\mathrm{HR}=0.47, p<0.0001$ ) (Figure $3(\mathrm{c})$ ) and expectoration (FAS: 4 days vs. 7 days, $H R=0.39, p<0.0001$ ) (Figure $3(d)$ ) were observed in the treatment group compared with the control group.

The overall symptom resolution rate at 28 days was higher in the treatment group in comparison with the control group (FAS: $83.33 \%$ vs. $68.06 \%, \mathrm{HR}=0.94$, $p=0.0326$ ) (Figure $4(\mathrm{a})$ ). The rate of recovery of chest CT signs also showed an increase in the treatment group (FAS: $86.11 \%$ vs. $72.22 \%, p=0.0402$ ) (Figure $4(\mathrm{~b})$ ). CT signs in two patients of the treatment group are shown in Figure 5. The CT results of patient 1 (Figure 5 (A1 and A2)) in the control group, to some extent, had a worse situation in lung tissue at day 12 compared with patient 2 in the treatment group (Figure 5 (B1 and B2)). More obvious consolidation of the left lower lobe and peripherals was observed in patient 1, while only slight consolidation of the right upper lobe in patient 2 was observed.

Taken together, LH tablets treatment resulted in an improved symptom resolution rate at 14 and 28 days, higher rate, and faster resolution of cough and expectoration and improved CT manifestations. 


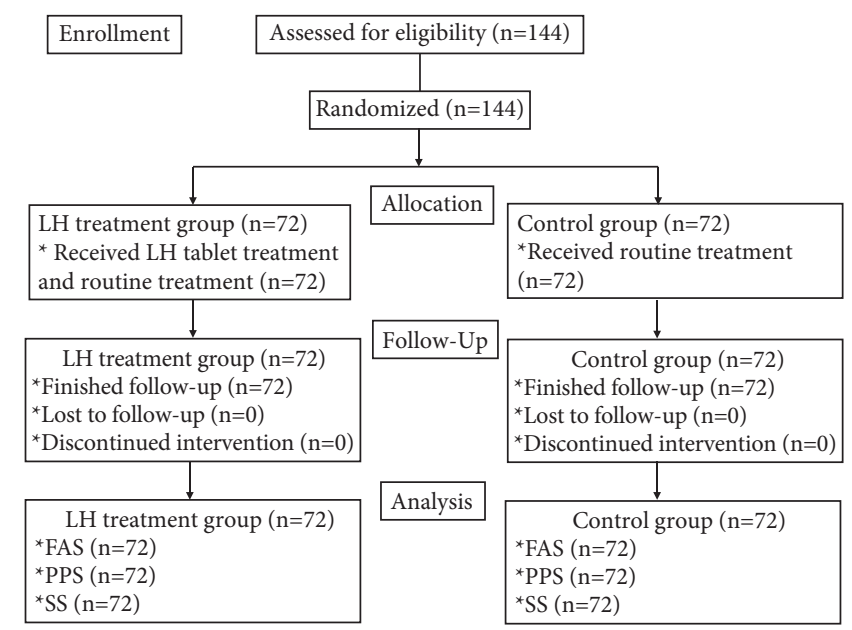

FIgURE 1: Study flowchart.

Table 1: Baseline demographic data.

\begin{tabular}{|c|c|c|c|}
\hline Variable & $\begin{array}{l}\text { Treatment group } \\
\qquad N=72\end{array}$ & $\begin{array}{c}\text { Control group } \\
\quad N=72\end{array}$ & $P$ value \\
\hline Age $(y, x \pm s)$ & $49.56 \pm 14.88$ & $52.81 \pm 14.83$ & 0.1915 \\
\hline Males $(N, \%)$ & $23(31.94 \%)$ & $24(33.33 \%)$ & 0.8589 \\
\hline Temperature $\left({ }^{\circ} \mathrm{C}, x \pm \mathrm{s}\right)$ & $37.14 \pm 0.81$ & $37.01 \pm 0.83$ & 0.2164 \\
\hline Respiratory rate $(\mathrm{bpm}, \mathrm{x} \pm \mathrm{s})$ & $19.75 \pm 2.45$ & $19.60 \pm 2.15$ & 0.6329 \\
\hline Pulse (times/min, $x \pm s$ ) & $79.72 \pm 10.21$ & $76.90 \pm 9.02$ & 0.3402 \\
\hline Systolic pressure $(\mathrm{mmHg}, x \pm s)$ & $124.75 \pm 16.30$ & $127.61 \pm 13.14$ & 0.0847 \\
\hline Diastolic pressure (mmHg, $x \pm s$ ) & $79.88 \pm 9.51$ & $80.53 \pm 10.29$ & 0.3990 \\
\hline \multicolumn{4}{|l|}{ Routine blood test } \\
\hline Leukocyte count $(N, x \pm s)$ & $5.09 \pm 1.79$ & $5.18 \pm 1.50$ & 0.5323 \\
\hline Neutrophil percentage $(\%, x \pm s)$ & $60.94 \pm 12.46$ & $61.22 \pm 13.26$ & 0.7064 \\
\hline Lymphocyte count $(N, x \pm s)$ & $1.49 \pm 0.60$ & $1.49 \pm 0.51$ & 0.5987 \\
\hline Concomitant medications & $56(78.87 \%)$ & $65(90.28 \%)$ & 0.0588 \\
\hline
\end{tabular}

$P$ value, comparison between the treatment group and the control group. $P<0.05$ indicates a significant difference.

TABle 2: Baseline clinical characteristics.

\begin{tabular}{lccc}
\hline Symptom & $\begin{array}{c}\text { Treatment } \\
\text { group } \\
N=72\end{array}$ & $\begin{array}{c}\text { Control } \\
\text { group } \\
N=72\end{array}$ & $P$ value \\
\hline Fever $(N, \%)$ & $34(47.22 \%)$ & $27(37.5 \%)$ & 0.3732 \\
Fatigue $(N, \%)$ & $20(27.78 \%)$ & $19(26.39 \%)$ & 0.8971 \\
Cough $(N, \%)$ & $64(89.89 \%)$ & $71(98.61 \%)$ & 0.4014 \\
Expectoration $(N, \%)$ & $29(40.28 \%)$ & $34(47.22 \%)$ & 0.7338 \\
Short breath $(N, \%)$ & $10(13.89 \%)$ & $8(11.11 \%)$ & 0.6232 \\
Chest tightness $(N, \%)$ & $11(15.28 \%)$ & $15(20.83 \%)$ & 0.3968 \\
Dyspnea $(N, \%)$ & $3(4.17 \%)$ & $3(4.17 \%)$ & 0.9908 \\
Headache $(N, \%)$ & $3(4.17 \%)$ & $3(4.17 \%)$ & 1.0000 \\
Nausea $(N, \%)$ & $5(6.94 \%)$ & $7(9.72 \%)$ & 0.5507 \\
Vomit $(N, \%)$ & $5(6.94 \%)$ & $5(6.94 \%)$ & 1.0000 \\
Diarrhea $(N, \%)$ & $4(5.56 \%)$ & $2(2.78 \%)$ & 0.4158 \\
Anorexia $(N, \%)$ & $6(8.33 \%)$ & $8(11.11 \%)$ & 0.5860 \\
Dry rale $(N, \%)$ & $0(0 \%)$ & $1(1.39 \%)$ & 0.3241 \\
Wet rale $(N, \%)$ & $1(1.39 \%)$ & $1(1.39 \%)$ & 1.0000 \\
\hline
\end{tabular}

$P$ value, comparison between the treatment group and the control group. $P<0.05$ indicates a significant difference.

3.4. Safety. Safety findings are given in Table 3. AEs' incidence rates were similar in both groups, with $9.72 \%$ and $15.28 \%$ in the LH group and control group, respectively $(p=0.3135)$. No death was observed in either group. Of the AEs occurring in the treatment group, 6 of the 7 cases involved had no relationship with LH tablet administration.

\section{Discussion}

COVID-19 has seriously impacted the whole world since it was first discovered in Wuhan, China [9, 10]. Although in some countries the first wave of the pandemic has been under control, the second or third wave is occurring or expected to happen. Due to the limited supply of effective vaccines [11], methods to reduce disease spread such as avoiding crowds, wearing masks, and keeping physical distance are still important strategies to fight this infection $[12,13]$. Similar to common cold and flu infections, coughing represents the main clinical sign of COVID-19 in its acute phase and continues in the postinfection phase [14]. Coughing not only brings pain but also promotes the spread of respiratory droplets in the community. Coughing patients may be stigmatized, leading to social isolation and widespread fear as a source of contagion, especially during the COVID-19 pandemic. Controlling COVID-19-related cough may reduce community transmission. 


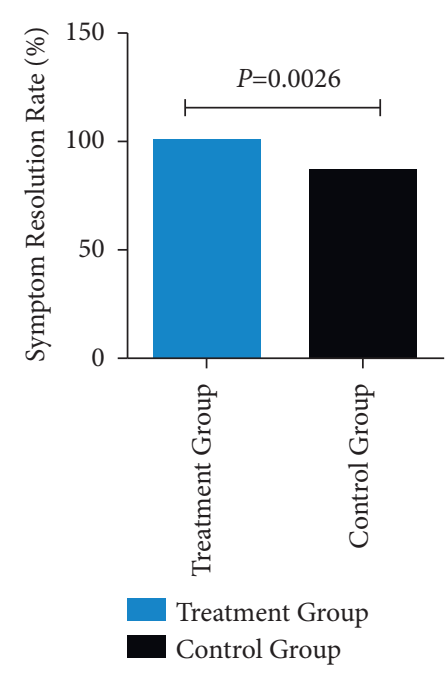

(a)

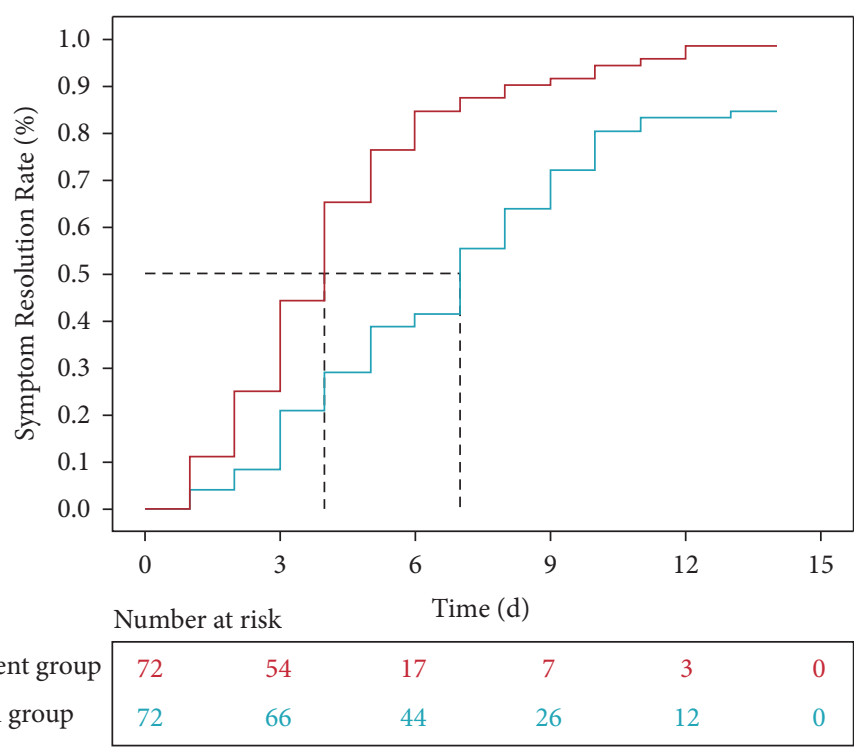

\begin{tabular}{lrrl} 
Group Event/total & Median(95\%CI) & HR(95\%CI) \\
Treatment & $71 / 72$ & $4.00(3.00-4.00)$ & $0.47(0.33-0.67)$ \\
Control & $61 / 72$ & $7.00(5.00-8.00)$ & Reference \\
\multicolumn{2}{c}{ Log-rank } & \multicolumn{2}{c}{$\mathrm{p}<0.0001$}
\end{tabular}

(b)

Figure 2: Rates of and times to symptom resolution.

Unmet clinical needs for cough treatment have been broadly documented. Not a few patients with coughing express concerns of cough treatment $[15,16]$. Up to now, treatment measures for acute and chronic cough in COVID19 follow recently published guidelines $[17,18]$. Although a few drugs for the relief of coughing are available on the market [19], there is still a lack of sound evidence demonstrating their therapeutic benefits for cough due to acute viral infections or pneumonia $[20,21]$. In the routine treatment proposed by the Diagnosis and Treatment Program for Novel Coronavirus Infection Pneumonia (Trial Eighth Edition), no medicine but respiratory support methods are recommended. Corticosteroids, prescriptions that are used in acute lower respiratory tract infection and post-COVID patients, showed no improved outcome in shortening coughing time [22]. Anti-inflammatory drugs such as dexamethasone, which decreases mortality in COVID-19, showed no confirmed curative effect in relieving coughing [23].

LH, mainly composed of Maxing Shigan and Qingjin Huatan decoctions, removes heat from the respiratory system and helps reduce sputum production and viscosity, promoting discharge in viral or bacterial infections. The clinical observation showed promising therapeutic effects in COVID-19 patients, especially concerning cough relief. However, high-level evidence is lacking. Therefore, this RCT was carried out to estimate the effectiveness of LH tablets in COVID-19. According to above results, LH treatment significantly improved the clinical symptom resolution rate at 14 days. In comparison with control cases, LH treatment resulted in decreased median time to cough and expectoration resolution. Besides, higher rates of chest CT manifestation improvement were observed after $\mathrm{LH}$ administration. In two cases of control and LH treatment groups at enrollment, obvious bilateral pulmonary infiltrates and ground glass could be observed in CT results. After 12day remedy, absorption of bilateral pulmonary infiltrates could be found in patients of both groups. However, consolidation of the left lower lobe and peripherals were observed in patients of the control group, while less consolidation of the right upper lobe was found in patients of the LH treatment group. However, LH treatment showed a limited synergistic effect in improving fever, chest tightness, and shortness of breath compared with the routine treatment. No differences were found in laboratory findings or viral burden. Seven cases in the treatment group and eleven cases in the control group were documented for adverse events. Gastrointestinal dysfunction and metabolic disorders were the most frequent adverse events according to organ classification. However, no severe events were documented and were definitely related to the treatment. No death event was reported.

Pharmacological research studies reported that the main ingredients of LH tablet may have antiviral and anti-inflammatory properties, contributing to the relief of respiratory symptoms and inflammatory responses. Dahuang and Gancao could suppress the membrane penetration of SARS$\mathrm{CoV}$ and inhibit the replication of SARS-CoV [24]. Jinyinhua and Lianqiao possess the inhibitory effect in excessive inflammatory responses $[25,26]$. Besides, it is documented that the most frequently used drug combination was Mahuang-Kuxingren, which contributes to the 


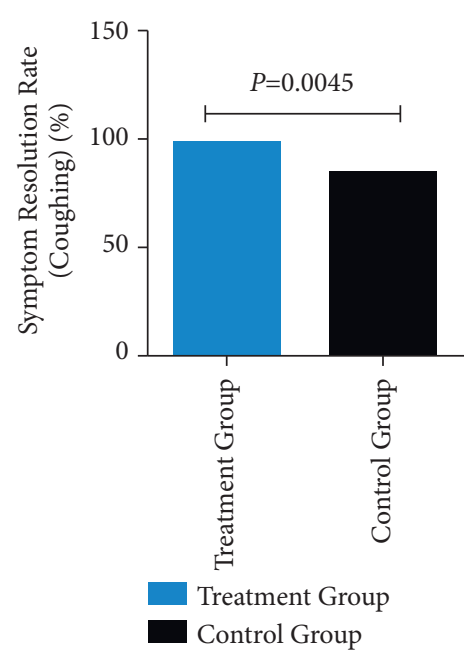

(a)

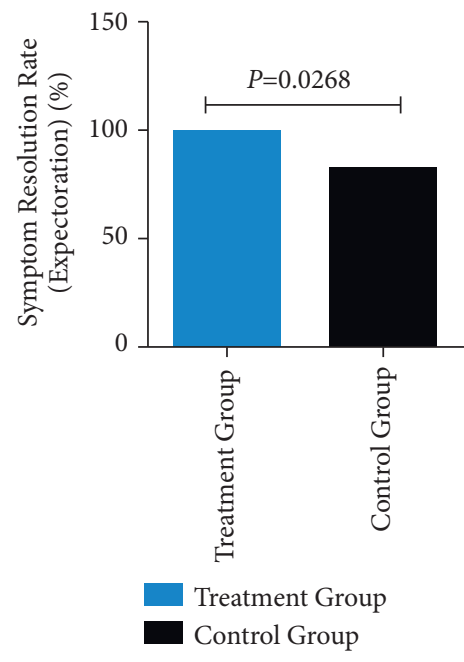

(c)

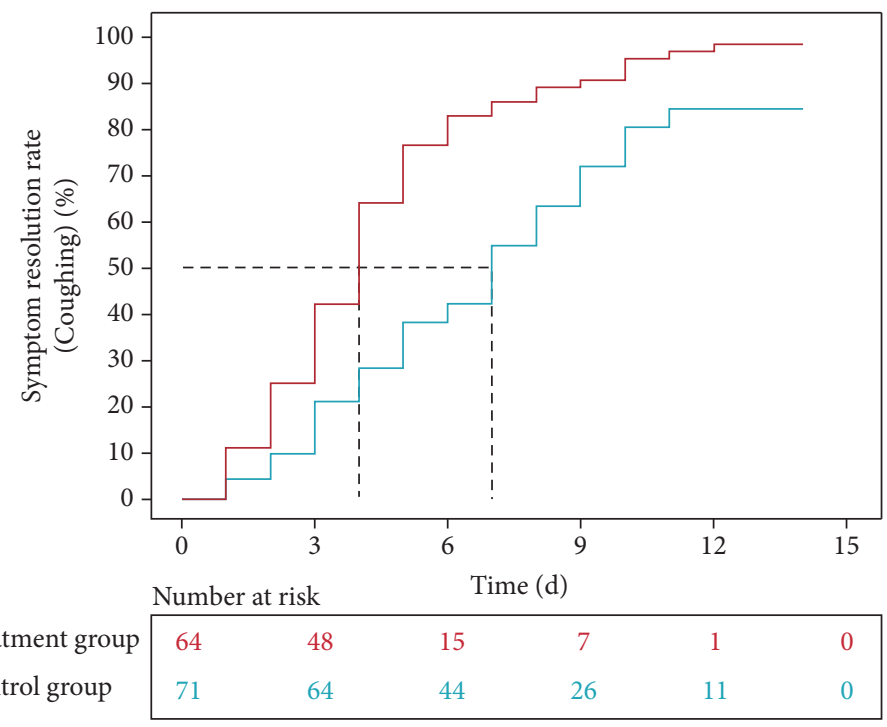

$\begin{array}{llll}\text { Treatment } & 63 / 64 & 4.00(3.00-4.00) & 0.48(0.38-0.70) \\ \text { Control } & 60 / 71 & 7.00(5.00-8.00) & \text { Reference }\end{array}$

Log-rank $\quad \mathrm{p}<0.0001$

(b)
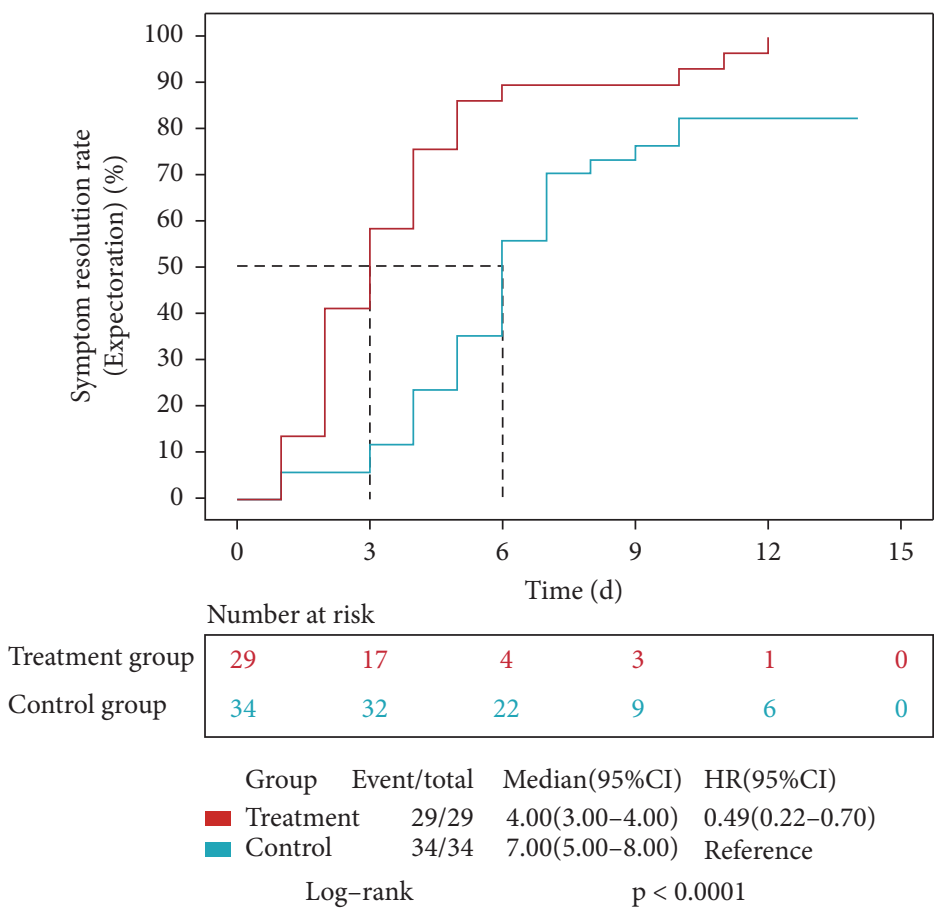

(d)

Figure 3: Rates of and times to respiratory symptom resolution.

shorter cough time and better CT outcome of COVID-19 patients [27]. However, more mechanism research studies are needed to reveal how LH tablets is beneficial for COVID19 patients.

There are some limitations in this study. The data to exhibit baseline clinical characteristics and adverse events were all enumeration data and only case number was recorded. No score methods were used for the evaluation of each symptom. Besides, 160 random numbers with block 4 were generated for the enrollment of patients in the beginning of this trial. Unexpectedly, the COVID-19 epidemic stopped before all 160 patients were included. However, 144 patients still met the need for our trial and followed the randomized principles. The baseline was also comparable. At 


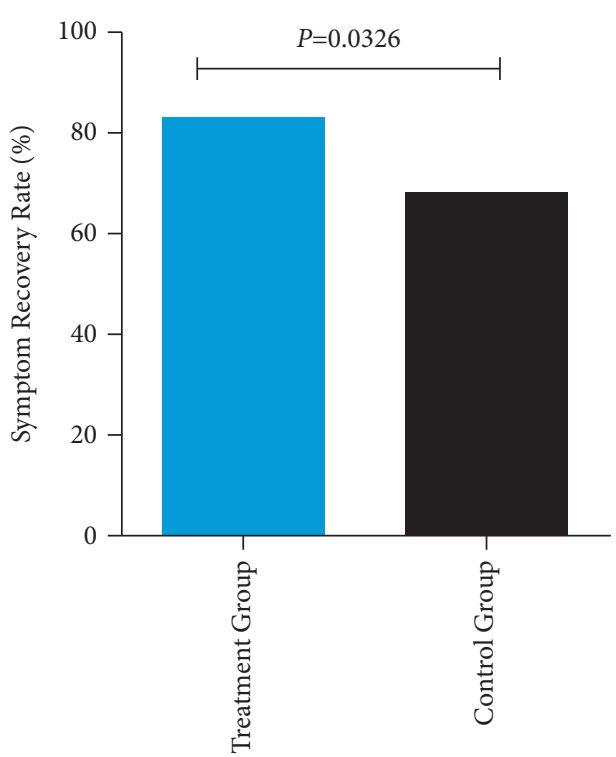

(a)

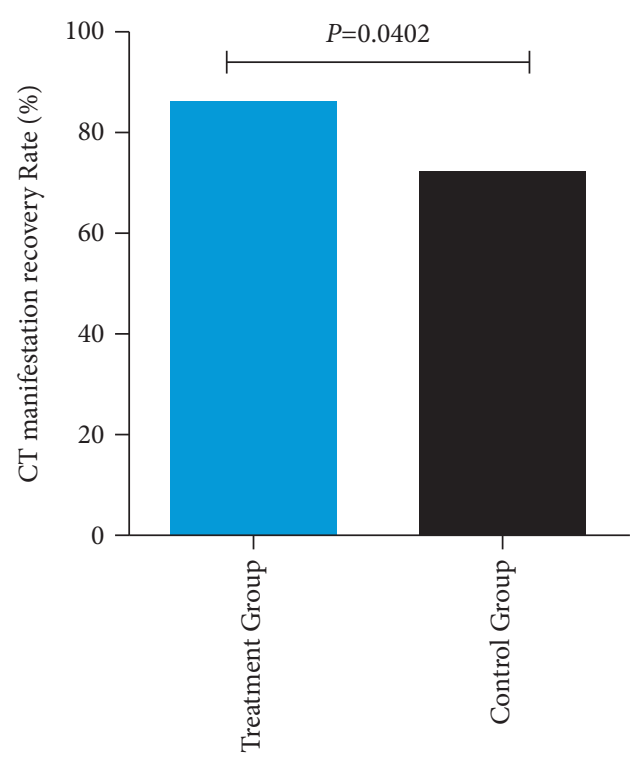

(b)

FIGURE 4: Rates of clinical cure and chest computed tomography manifestation improvement.
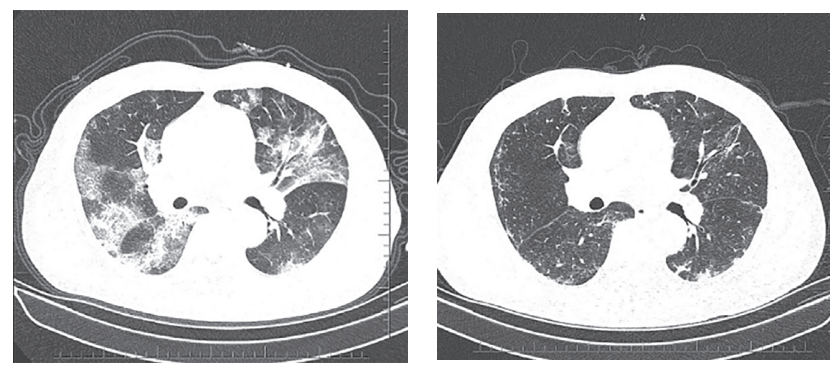

(a)
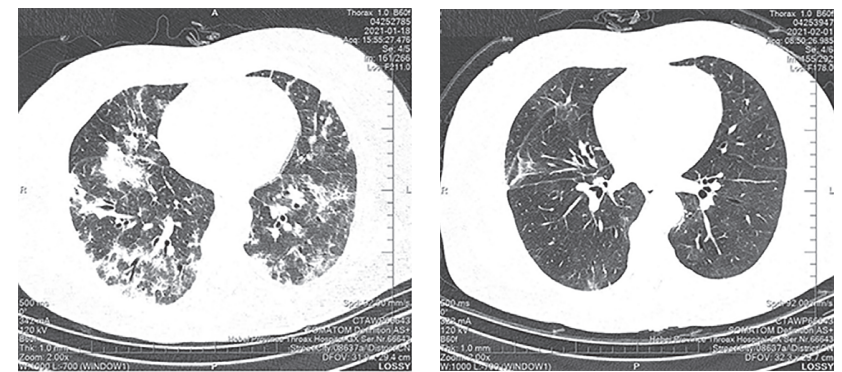

(b)

Figure 5: Computed tomography (CT) manifestations at enrolment and post-treatment.

TABle 3: Adverse events.

\begin{tabular}{|c|c|c|c|}
\hline Adverse event & $\begin{array}{l}\text { Treatment group } \\
\qquad N=72\end{array}$ & $\begin{array}{c}\text { Control group } \\
\quad N=72\end{array}$ & $P$ value \\
\hline Total $(N, \%)$ & $7(9.72 \%)$ & $11(15.28 \%)$ & 0.3135 \\
\hline Heart dysfunction $(N, \%)$ & $0(0 \%)$ & $1(1.39 \%)$ & \\
\hline Gastrointestinal dysfunction $(N, \%)$ & $1(1.39 \%)$ & $6(8.33 \%)$ & \\
\hline Hepatobiliary dysfunction $(N, \%)$ & $1(1.39 \%)$ & $1(1.39 \%)$ & \\
\hline Infections $(N, \%)$ & $1(1.39 \%)$ & $1(1.39 \%)$ & \\
\hline Metabolic disorders $(N, \%)$ & $3(4.17 \%)$ & $2(2.78 \%)$ & \\
\hline Neurological disorders $(N, \%)$ & $1(1.39 \%)$ & $0(0 \%)$ & \\
\hline Severity ${ }^{[1]}$ & & & 0.3889 \\
\hline Mild $(N, \%)$ & $6(8.33)$ & $11(15.28)$ & \\
\hline Moderate $(N, \%)$ & $1(1.39)$ & $0(0.00)$ & \\
\hline Severe $(N, \%)$ & $0(0.00)$ & $0(0.00)$ & \\
\hline Related to treatment & & & 0.7484 \\
\hline Definitely related & $0(0.00)$ & $0(0.00)$ & \\
\hline Probably related & $0(0.00)$ & $0(0.00)$ & \\
\hline Definitely not related & $1(1.39)$ & $1(1.39)$ & \\
\hline Probably not related & $6(8.33)$ & $8(11.11)$ & \\
\hline Not sure & $0(0.00)$ & $2(2.78)$ & \\
\hline Death $(N, \%)$ & $0(0.00)$ & $0(0.00)$ & 1.0000 \\
\hline
\end{tabular}


last, we have to stress that all data from patients are recorded at day one of the enrollment, not the first time that disease occurred.

Overall, this RCT suggested LH tablets might be beneficial to symptomatic COVID-19 cases, especially in relieving cough and expectoration.

$\begin{array}{ll}\text { Abbreviations } \\ \text { COVID-19: } & \text { Coronavirus disease } 2019 \\ \text { LH: } & \text { Lianhua Qingke } \\ \text { SARS- } & \text { Severe acute respiratory syndrome } \\ \text { CoV-2: } & \text { coronavirus 2 } \\ \text { WHO: } & \text { World Health Organization } \\ \text { AEs: } & \text { Adverse events } \\ \text { CT: } & \text { Computed tomography } \\ \text { FAS: } & \text { Full analysis set } \\ \text { PPS: } & \text { Per protocol set } \\ \text { SS: } & \text { Safety set. }\end{array}$

\section{Data Availability}

The datasets used and/or analyzed in this study are available from the corresponding author upon request.

\section{Ethical Approval}

The study followed the GCP guidelines and the Declaration of Helsinki (as revised in 2013). This study had approval from the Ethics Committee of Hebei Chest Hospital (20372504D) and was registered in Chinese Clinical Trial Registry (ChiCTR2000029589).

\section{Consent}

The publication of CT images was approved by the patients. Signed informed consent was provided by each patient or their legal representative.

\section{Conflicts of Interest}

The authors declare that they have no conflicts of interest.

\section{Authors' Contributions}

Ling Zhang, Lei $\mathrm{Wu}$, and Xiaolong Xu contributed equally to this study. LQQ, MJQ, and WSC designed the trial and provided administrative support. ZL, WL, XXL, YYD, JMR, YXX, ZX, GY, SHX, HJ, and LB provided study materials and collected patient information. ZL, WL, and XXL analyzed and interpreted the data and were major contributors in writing the manuscript. All authors read and approved the final manuscript.

\section{Acknowledgments}

The present work was supported by the Key Research and Development Plan of Hebei Province (20372504D), the Special Project of Ministry of Science and Technology of China (2020YFC0841600), and the National Major Scientific and Technological Project (2017ZX10305501). Statistical analysis was performed by the School of Public Health, Tongji Medical College, Huazhong University of Science and Technology.

\section{Supplementary Materials}

Table S1. Routine treatment in each group. CONSORT 2010 checklist of information to include when reporting a randomised trial. (Supplementary Materials)

\section{References}

[1] J. G. Adams and R. M. Walls, "Supporting the health care workforce during the COVID-19 global epidemic," JAMA, vol. 323, no. 15, pp. 1439-1440, 2020.

[2] S. M. Kissler, C. Tedijanto, E. Goldstein, Y. H. Grad, and M. Lipsitch, "Projecting the transmission dynamics of SARSCoV-2 through the postpandemic period," Science, vol. 368, no. 6493 , pp. 860-868, 2020.

[3] Z. Khan, Y. Karatas, and H. Rahman, "Anti COVID-19 drugs: need for more clinical evidence and global action," Advances in Therapy, vol. 37, no. 6, pp. 2575-2579, 2020.

[4] W. J. Wiersinga, A. Rhodes, A. C. Cheng, S. J. Peacock, and H. C. Prescott, "Pathophysiology, transmission, diagnosis, and treatment of Coronavirus Disease 2019 (COVID-19): a review," JAMA, vol. 324, no. 8, pp. 782-793, 2020.

[5] X. Li, L. Wang, S. Yan et al., "Clinical characteristics of 25 death cases with COVID-19: a retrospective review of medical records in a single medical center, Wuhan, China," International Journal of Infectious Diseases, vol. 94, pp. 128-132, 2020.

[6] W. J. Song, C. Hui, J. H. Hull et al., "Confronting COVID-19associated cough and the post-COVID syndrome: role of viral neurotropism, neuroinflammation, and neuroimmune responses," The Lancet Respiratory Medicine, vol. 9, no. 5, pp. 533-544, 2021.

[7] H. K. Won and W. J. Song, "Impact and disease burden of chronic cough," Asia Pacific Allergy, vol. 11, no. 2, p. e22, 2021.

[8] L. R. Jin, Y. Xu, and H. Yuan, "Effects of four types of integrated Chinese and Western medicines for the treatment of COVID-19 in China: a network meta-analysis," Revista da Associação Médica Brasileira (1992), vol. 66, no. 6, pp. 771-777, 2020.

[9] C. Huang, Y. Wang, X. Li et al., "Clinical features of patients infected with 2019 Novel coronavirus in Wuhan, China," The Lancet, vol. 395, no. 10223, pp. 497-506, 2020.

[10] F. Zhou, T. Yu, R. Du et al., "Clinical course and risk factors for mortality of adult inpatients with COVID-19 in Wuhan, China: a retrospective cohort study," The Lancet, vol. 395, no. 10229, pp. 1054-1062, 2020.

[11] K. M. Bubar, K. Reinholt, S. M. Kissler et al., "Model-informed COVID-19 vaccine prioritization strategies by age and serostatus," Science, vol. 371, no. 6532, pp. 916-921, 2021.

[12] E. Rosenstrom, B. E. Oruc, N. Hupert et al., "High-quality masks reduce COVID-19 infections and deaths in the US," 2021.

[13] S. Park, B. Kim, and J. Lee, "Social distancing and outdoor physical activity during the COVID-19 outbreak in South Korea: implications for physical distancing strategies," Asia-Pacific Journal of Public Health, vol. 32, no. 6-7, pp. 360-362, 2020.

[14] R. Dhand and J. Li, "Coughs and sneezes: their role in transmission of respiratory viral infections, including SARSCoV-2," American Journal of Respiratory and Critical Care Medicine, vol. 202, no. 5, pp. 651-659, 2020. 
[15] S.-Y. Kang, H.-K. Won, S. M. Lee et al., "Impact of cough and unmet needs in chronic cough: a survey of patients in Korea," Lung, vol. 197, no. 5, pp. 635-639, 2019.

[16] S. A. F. Chamberlain, R. Garrod, A. Douiri et al., "The impact of chronic cough: a cross-sectional European survey," Lung, vol. 193, no. 3, pp. 401-408, 2015.

[17] A. H. Morice, E. Millqvist, K. Bieksiene, S. S. Birring, P. Dicpinigaitis, and R. C. Domingo, "ERS guidelines on the Diagnosis and treatment of chronic cough in adults and children," European Respiratory Journal, vol. 55, no. 1, 2020.

[18] P. Gibson, G. Wang, L. McGarvey et al., "Treatment of unexplained chronic cough," Chest, vol. 149, no. 1, pp. 27-44, 2016.

[19] S. B. Mazzone and L. McGarvey, "Mechanisms and rationale for targeted therapies in refractory and unexplained chronic cough," Clinical Pharmacology \& Therapeutics, vol. 109, no. 3, pp. 619-636, 2021.

[20] C. C. Chang, A. C. Cheng, and A. B. Chang, "Over-thecounter (OTC) medications to reduce cough as an adjunct to antibiotics for acute pneumonia in children and adults," Cochrane Database of Systematic Reviews, vol. 3, Article ID CD006088, 2014.

[21] K. Schroeder and T. Fahey, "Systematic Review of randomised controlled trials of over the counter cough medicines for acute cough in adults," BMJ, vol. 324, no. 7333, p. 329, 2002.

[22] A. D. Hay, P. Little, A. Harnden et al., "Effect of oral prednisolone on symptom duration and severity in nonasthmatic adults with acute lower respiratory tract infection," JAMA, vol. 318, no. 8, pp. 721-730, 2017.

[23] H. W. Lee, J. Park, J. K. Lee, T. Y. Park, and E. Y. Heo, “The effect of the timing of dexamethasone administration in patients with COVID-19 pneumonia," Tuberculosis and Respiratory Diseases, vol. 84, no. 3, pp. 217-225, 2021.

[24] H. Yan and C. Zou, "Mechanism and material basis of lianhua qingwen capsule for improving clinical cure rate of COVID19: a study based on network pharmacology and molecular docking technology," Nan Fang Yi Ke Da Xue Xue Bao, vol. 41, no. 1, pp. 20-30, 2021.

[25] L. Gong, L. Yu, X. Gong et al., "Exploration of anti-inflammatory mechanism of forsythiaside a and forsythiaside $\mathrm{B}$ in $\mathrm{CuSO}_{4}$-induced inflammation in zebrafish by metabolomic and proteomic analyses," Journal of Neuroinflammation, vol. 17, no. 1, p. 173, 2020.

[26] Y. Li, W. Li, C. Fu, Y. Song, and Q. Fu, "Lonicerae japonicae flos and lonicerae flos: a systematic review of ethnopharmacology, phytochemistry and pharmacology," Phytochemistry Reviews, vol. 19, no. 1, pp. 1-61, 2019.

[27] Z. Zhou, N. Gao, Y. Wang, P. Chang, Y. Tong, and S. Fu, "Clinical studies on the treatment of novel coronavirus pneumonia with traditional Chinese medicine-a literature analysis," Frontiers in Pharmacology, vol. 11, Article ID 560448, 2020. 